\title{
Data compression in machine learning applied to natural language
}

\author{
JERZY W. GRZYMALA-BUSSE and SOE THAN \\ University of Kansas, Lawrence, Kansas
}

\begin{abstract}
In this paper, we investigate the possibility of applying machine learning methods to data derived from the area of natural language and show how rules, induced by machine learning, are changed after the original data are compressed by grouping together entries, attributes, and attribute values. Also shown is how excessive compression of input data may affect the accuracy of induced rules.
\end{abstract}

It is becoming more and more clear that it is possible to efficiently study the semantic space of an entire natural language. A conceptual dictionary, Roget's International Thesaurus, is a good approximation of the semantic space of the English language (Sedelow \& Sedelow, 1992). An effort to show the adequacy of Roget's International Thesaurus to this purpose was successful (e.g., Brady, 1991, personal communication, December 29, 1991; Old, 1991).

The main aim of this paper is to show that machine learning can be successfully used to study Roget's International Thesaurus as well. Another aim is to show how compression of original data may affect rules induced by machine learning methods.

The basic assumption of the present paper is that the input data for machine learning are stored in a decision table. Each entry of the decision table is described by attributes and a decision. An entry from the decision table is an example from the viewpoint of machine learning. For example, entries may be words in Roget's International Thesaurus that are in the same paragraphs with the word over. Attributes are: spelling of the word, part of speech, and Bryan Type 10 component number. The decision is the category.paragraph.semicolon location of the word in the thesaurus. Such a file, consisting of 400 entries, was presented by J. Brady (personal communication, December 29, 1991). Machine learning algorithms induce rules to identify the decision values when the values of attributes are known. The present paper shows how such rules are changed after the decision table is compressed, which is accomplished by grouping together entries, attributes, and attribute values.

The operation of compression may be applied before the main process of learning, so it is a kind of preprocessing. Among other preprocessing techniques used in machine learning are selecting the most representative examples (Kibler \& Aha, 1987; Michalski \& Chilausky, 1980) and approaches to reduction of input data (Pawlak, Slowinski, \& Slowinski, 1986; Van de Velde, 1988).

Address correspondence to J. Grzymala-Busse, Department of Computer Science, University of Kansas, Lawrence, KS 66045 (e-mail: jerzy@hawk.cs.ukans.edu).
The present paper continues the work of Grzymala-Busse (1989), in which the compression of input data was accomplished by grouping together examples, attributes, and tuples of attribute values into blocks such that blocks of examples and attributes were transformed into blocks of tuples of attribute values in the same way that their members were transformed originally. Grzymala-Busse introduced the notion of a partition triple, consisting of a partition on the set of all examples, a partition on the set of all attributes, and a partition on the set of tuples of attribute values, where each tuple of attribute values is constructed from values of a single block of attributes, and each such block is a member of the partition on the set of all attributes. One of the assumptions of GrzymalaBusse was that the partition on the set of all attributes is fixed. Grzymala-Busse and Than (1992) assumed that the partition on the set of all attributes is not fixed. The compressed decision table is simpler, while the accuracy of rules induced from the compressed decision table by the learning system may be reduced. Similar problems were considered by Pawlak, Slowinski, and Slowinski (1986), who found that overgeneralization was useful. In general, overgeneralization may result in much simpler rules at little expense to accuracy.

\section{DECISION TABLES AND MACHINE LEARNING}

The fundamental assumption of the present paper is that the input data for a learning system are collected in a decision table. The following definition of a decision table is a slightly modified version of the definition introduced by Pawlak (1982). The decision table $S$ is a sextuple ( $E$, $A, V, d, W, \rho)$, where

$E$ is a finite nonempty set of examples,

$A$ is a finite nonempty set of attributes,

$V$ is a finite nonempty set of attribute values,

$d$ is a variable called a decision,

$W$ is a finite nonempty set of decision values,

$\rho: E \times[A \cup\{d\}] \rightarrow V \cup W$, where if $\rho$ is restricted to $E \times A$, it has values from $V$, and if $\rho$ is restricted to $E \times\{d\}$, it has values from $W$. 
For the sake of simplicity, restrictions of $\rho$ to $E \times A$ and to $E \times\{d\}$ also will be denoted by $\rho$.

The decision table $S=(E, A, V, d, W, \rho)$ will be called consistent if, and only if, for any two examples, characterized by equal values for any attribute, their decision values are also equal. Obviously, in the case of consistent decision tables, each value of the expert's decision $d$ may be recognized on the basis of values of attributes.

Machine learning is based on discovering similarities between positive examples representing the same concept, and dissimilarities between positive and negative examples representing other concepts. The goal of machine learning is to find a description of the concept-to describe all positive examples from the concept and no negative examples. Such a description may be expressed, for example, by a set of rules or a decision tree.

An example of the decision table is given in Table 1, which presents 19 words from Roget's International Thesaurus that are in the same paragraphs with the word over-a small subset of the file from Brady (personal communication, December 29, 1991), selected here for an illustration. Attributes are: Spelling _ of the _word, Part_of_speech, Bryan_Type_10_ component number, coded as Spelling, Part_of_Speech, Type_10, respectively, and a decision is the category.paragraph.semicolon location of the entry in the thesaurus, coded as Location.

The data, presented in Table 1, were processed by the program LERS (Learning from Examples based on Rough Sets) implemented on VAX 9000 at the University of Kansas. The following rules were induced by the single local covering option of the LERS system (GrzymalaBusse, 1989):

(Type_10, 6186) $\rightarrow$ (Location, 0036.13.001),

(Part_of_Speech, ADJS) \& (Type_10, 222) (Location, 0036.13.001),

(Part_of_Speech, PREPS) $\rightarrow$ (Location, 0036.20.001), (Part of Speech, ADVS) $\rightarrow$ (Location, 0040.10.001), (Type_10, 7343) $\rightarrow$ (Location, 0043.06.001),

Table 1

\begin{tabular}{rlrrc}
\hline & Spelling & Part_of_Speech & Type_10 & Location \\
\hline 1 & superior & ADJS & 6186 & 0036.13 .001 \\
2 & greater & ADJS & 6186 & 0036.13 .001 \\
3 & higher & ADJS & 6186 & 0036.13 .001 \\
4 & over & ADJS & 222 & 0036.13 .001 \\
5 & above & ADJS & 222 & 0036.13 .001 \\
6 & beyond & PREPS & 222 & 0036.20 .001 \\
7 & over & PREPS & 222 & 0036.20 .001 \\
8 & past & PREPS & 222 & 0036.20 .001 \\
9 & over & ADVS & 222 & 0040.10 .001 \\
10 & above & ADVS & 222 & 0040.10 .001 \\
11 & beyond & ADVS & 222 & 0040.10 .001 \\
12 & remaining & ADJS & 7343 & 0043.06 .001 \\
13 & leftover & ADJS & 7343 & 0043.06 .001 \\
14 & completeness & NOUNS & 3270 & 0056.01 .001 \\
15 & wholeness & NOUNS & 3270 & 0056.01 .001 \\
16 & entireness & NOUNS & 3270 & 0056.01 .001 \\
17 & end & VERBS & 1851 & 0070.05 .001 \\
18 & terminate & VERBS & 1851 & 0070.05 .001 \\
19 & conclude & VERBS & 1851 & 0070.05 .001 \\
\hline
\end{tabular}

(Part_of_Speech, NOUNS) $\rightarrow$ (Location, 0056.01.001), (Par_ of_Speech, VERBS) $\rightarrow$ (Location, 0070.05.001).

\section{PARTITION TRIPLES AND COMPRESSION OF DECISION TABLES}

The idea of compressing data was originally developed in automata theory, and was termed partition pairs (see, e.g., Hartmanis \& Stearns, 1966, chap. 3). This idea was extended to input data of machine learning systems by Grzymala-Busse (1990). In Grzymala-Busse and Than (1992), Grzymala-Busse's (1990) idea of a partition triple was generalized; the partition on the set of all attributes is no longer fixed and, as a result, the partition on the set of values is defined in a simpler way.

Let $X$ be a nonempty finite set. A partition $\pi$ on $X$ is a family of disjoint subsets of $X$ whose set union is $X$. Elements of partition $\pi$ will be called blocks of $\pi$. If elements $x$ and $y$ are both members of the same block of $\pi$, it will be denoted by $x \equiv y(\pi)$. There are two trivial partitions $0_{x}$ and $1_{x}$, where $0_{x}$ is the partition on $X$ in which all blocks are one-element subsets of $X$ and $1_{x}$ is the partition on $X$ that contains only one block.

For a decision table $(E, A, V, d, W, \rho)$, let $\pi$ be a partition on $E$, let $\tau$ be a partition on $A$, and let $\lambda$ be a partition on $V$. A partition triple on a decision table $S=(E, A$, $V, d, W, \rho)$ is an ordered triple of partitions $(\pi, \tau, \lambda)$ such that for all $x, y \in E$ and $a, b \in A$

$$
\begin{aligned}
& x \equiv y(\pi) \text {, and } a \equiv b(\tau) \text { implies that } \\
& \qquad \rho(x, a) \equiv \rho(y, b)(\lambda) \text { and } \rho(x, d)=\rho(y, d) .
\end{aligned}
$$

Partition triples may be used to construct simpler, compressed decision tables, as shown in the following definition.

Let $(\pi, \tau, \lambda)$ be a partition triple on a decision table $S=(E, A, V, d, W, \rho)$. The $(\pi, \tau, \lambda)$ image of $S$ is the decision table $\left(\pi, \tau, \lambda, d, W, \rho^{\prime}\right)$ such that for all $B_{\pi} \in \pi$, $B_{\tau} \in \tau$, and $B_{\lambda} \in \lambda$

$$
\rho^{\prime}\left(B_{\pi}, B_{\tau}\right)=B_{\lambda} \text { and } \rho^{\prime}\left(B_{\pi}, d\right)=\rho(x, d)
$$

if, and only if,

$$
\rho(x, a)=v,
$$

where $x, a$, and $v$ are arbitrary members of $B_{\pi}, B_{\tau}$, and $B_{\lambda}$, respectively.

A method for generating partition triples employs a special kind of partition triple, called $M M m$ triples (see Grzymala-Busse \& Than, 1992).

For the decision table $S=(E, A, V, d, W, \rho)$ from Table 1, and the partitions

$$
\begin{aligned}
& \pi=\{\{1,2,3\},\{4,5\},\{6,7\},\{8\},\{9,10,11\}, \\
& \{12,13\},\{14,15,16\},\{17,18,19\}\} \text {, } \\
& \tau=0_{A},
\end{aligned}
$$

and

$$
\begin{aligned}
& \lambda=\{\text { superior, } \text { greater, higher }\}, \\
& \text { \{over, above, beyond }\},\{\text { past }\} \text {, } \\
& \text { \{remaining, leftover\}, }
\end{aligned}
$$


\{completeness, wholeness, entireness\},

\{end, terminate, conclude $\},$ ADJS $\}$,

\{PREPS $\},\{$ ADVS $\},\{$ NOUNS $\},\{$ VERBS $\}$, $\{6186\},\{222\},\{7343\},\{3270\},\{1851\}\}$,

$(\pi, \tau, \lambda)$ is a partition triple on $S$. The $(\pi, \tau, \lambda)$ image of the decision table from Table 1 is presented in Table 2 , where examples are renumbered, and blocks \{superior, greater, higher $\},\{$ over, above, beyond $\},\{$ past $\},\{$ remaining, leftover $\}$, \{completeness, wholeness, entireness $\}$, and $\{$ end, terminate, conclude $\}$ are coded as SUPERIOR, OVER, PAST, REMAINING, COMPLETENESS, and END, respectively. The other singleton blocks are coded as to their respective contents.

The following rules were induced by the LERS option single local covering:

(Spelling, SUPERIOR) $\rightarrow$ (Location, 0036.13.001),

(Spelling, OVER) \& (Par__of_Speech, ADJS) $\rightarrow$ (Location, 0036.13.001),

(Part_of_Speech, PREPS) $\rightarrow$ (Location, 0036.20.001),

(Part_of Speech, ADVS) $\rightarrow$ (Location, 0040.10.001),

(Spelling, REMAINING) $\rightarrow$ (Location, 0043.06.001),

(Spelling, COMPLETENESS) $\rightarrow$ (Location, 0056.01.001),

(Spelling, END) $\rightarrow$ (Location, 0070.05.001).

It is clear that the two sets of rules, induced from Tables 1 and 2 , are different. Although both decision tables, presented as Tables 1 and 2, use the same set of attributes, the induced rules use different attributes. For example, Spelling was not used in the first set of rules at all.

Here, the rules of the form (Spelling, SUPERIOR) $\rightarrow$ (Location, 0036.13.001) and (Part_of_Speech, PREPS) $\rightarrow$ (Location, 0036.20.001) look unrealistic, but it is because of the small set of data in this particular example.

\section{OVERGENERALIZATION}

The decision table from Table 2 , a $(\pi, \tau, \lambda)$ image of the decision table from Table 1 , as well as the original decision table, is consistent. However, in general, a ( $\pi$, $\tau, \lambda$ ) image of a decision table $S$ does not need to be consistent, even when the original decision table $S$ is consistent. The following example supports that claim.

Let $\pi, \tau$, and $\lambda$ be the following partitions on the sets of examples, attributes, and values of the decision table $S$ from Table 1:

$$
\begin{gathered}
\pi=\{\{1,2,3\},\{4,5\},\{6,7\},\{8\},\{9,10,11\}, \\
\{12,13\},\{14,15,16\},\{17,18,19\}\},
\end{gathered}
$$

Table 2

\begin{tabular}{lllrr}
\hline \multicolumn{1}{c}{ Spelling } & Part_of_Speech & Type_10 & Location \\
\hline 1 & SUPERIOR & ADJS & 6186 & 0036.13 .001 \\
2 & OVER & ADJS & 222 & 0036.13 .001 \\
3 & OVER & PREPS & 222 & 0036.20 .001 \\
4 & PAST & PREPS & 222 & 0036.20 .001 \\
5 & OVER & ADVS & 222 & 0040.10 .001 \\
6 & REMAINING & ADJS & 7343 & 0043.06 .001 \\
7 & COMPLETENESS & NOUNS & 3270 & 0056.01 .001 \\
8 & END & VERBS & 1851 & 0070.05 .001 \\
\hline
\end{tabular}

Table 3

\begin{tabular}{llccc}
\hline & \multicolumn{1}{c}{ Spelling } & Part_of_Speech_Type_10 & Location \\
\hline 1 & SUPERIOR & JPV_6186_222_3270 & 0036.13 .001 \\
2 & OVER & JPV_6186_222_3270 & 0036.13 .001 \\
3 & OVER & JPV_6186_222_3270 & 0036.20 .001 \\
4 & PAST & JPV_6186_222_3270 & 0036.20 .001 \\
5 & OVER & JPV_6186_222_3270 & 0040.10 .001 \\
6 & REMAINING & JPV_6186_222_3270 & 0043.06 .001 \\
7 & COMPLETENESS & N_3270 & 0056.01 .001 \\
8 & END & V__1851 & 0070.05 .001 \\
\hline
\end{tabular}

$$
\tau=\{\{\text { Spelling }\},\{\text { Part_of_Speech, Type_10 }\}\},
$$

and

$$
\begin{aligned}
& \lambda=\{\{\text { superior, greater, higher }\}, \\
& \text { \{over, above, beyond\}, \{past\}, } \\
& \text { \{remaining, leftover\}, } \\
& \text { \{completeness, wholeness, entireness\}, } \\
& \text { \{end, terminate, conclude\}, } \\
& \text { \{ADJS, PREPS, ADVS, 6186, 222, 7343\}, } \\
& \text { \{NOUNS, 3270\}, \{VERBS, 1851\}\}. }
\end{aligned}
$$

$\{\pi, \tau, \lambda\}$ is a partition triple on $S$. The $\{\pi, \tau, \lambda\}$ image of the decision table from Table 1 is presented in Table 3 , where examples are renumbered, and blocks \{superior, greater, higher $\},\{$ over, above, beyond $\},\{$ past $\},\{$ remaining, leftover $\}$, \{completeness, wholeness, entireness \}, \{end, terminate, conclude\}, \{ADJS, PREPS, ADVS, 6186, 222, 7343\}, \{NOUNS, 3270\}, and \{VERBS, 1851\} are coded as SUPERIOR, OVER, PAST, REMAINING, COMPLETENESS, END, JPV_6186_222_3270, N_3270, and V_1851, respectively.

The decision table, presented in Table 3 , is not consistent. Using the algorithm LERS, it is possible to induce certain rules based on lower approximations of respective concepts. The notion of a lower approximation of a subset of $E$ was introduced in Pawlak (1982) as the rough set theory. The following rules were induced by the same single local covering option of the algorithm LERS:

(Spelling, SUPERIOR)-(Location, 0036.13.001), (Spelling, PAST) $\rightarrow$ (Location, 0036.20.001),

(Spelling, REMAINING) $\rightarrow$ (Location, 0043.06.001), (Spelling, COMPLETENESS) $\rightarrow$ (Location, 0056.01.001), (Spelling, END) $\rightarrow$ (Location, 0070.05.001).

The accuracy of the set of rules induced from the decision table from Table 3, compared with the original decision table represented by Table 1 , is equal to the ratio of the cardinality of the union of lower approximations of all concepts to the cardinality of the original example set, that is, $12 / 19 \approx 63 \%$. Thus the accuracy is lower, while induced rules are much simpler.

\section{CONCLUSIONS}

The present paper shows how machine learning methods may be used for studying natural language, on the basis of a subset of the file of 400 words in Roget's International Thesaurus that are in the same paragraphs with 
the word over. Obviously, machine learning methods can be used for the entire file. When that is done, two sets of rules are induced-the set of 254 certain rules and the set of 298 possible rules. Induction of two sets of rules is caused by inconsistencies in the original file. Rules induced from the smaller decision tables, obtained as a result of compression, may be simpler. Excessive use of compression may lead to overgeneralization; that is, the new decision tables may no longer be consistent, even when the original decision table was consistent. However, the accuracy of the simpler set of rules still may be sufficient.

\section{REFERENCES}

BradY, J. (1991). Towards automatic categorization of concordances using Roget's International Thesaurus. In Proceedings of the Third Midwest Artificial Intelligence and Cognitive Science Society Conference (pp. 93-97). Carbondale, IL.

GrZymala-BusSE, J. W. (1989). An overview of the LERS1 leaming system. In Proceedings of the Second International Conference on Industrial and Engineering Applications of Artificial Intelligence and Expert Systems (pp. 838-844). New York: ACM Press.

Grzymala-Busse, J. W. (1990). On the reduction of instance space in learning from examples. In Proceedings of the Fifth International Symposium on Methodologies for Intelligent Systems (pp. 388-395). Amsterdam: North-Holland.
Grzymala-Busse, J. W., \& Than, S. (1992). On the compression of instance space in inductive learning. In Proceedings of the Fourth Midwest Artificial Intelligence and Cognitive Science Society Conference (pp. 92-96), Utica, IL.

Hartmanis, J., \& STEARNs, R. E. (1966). Algebraic structure theory of sequential machines. Englewood Cliffs, NJ: Prentice-Hall.

KuBleR, D., \& AHA, D. W. (1987). Learning representative exemplars of concepts: An initial case study. In Proceedings of the Fourth International Workshop on Machine Leaming (pp. 24-30). Los Altos, CA: Morgan Kaufmann

Michalski, R. S., \& Chilausky, R. L. (1980). Knowledge acquisition by encoding expert rules versus computer induction from examples: A case study involving soybean pathology. Intermational Journal of Man-Machine Studies, 12, 63-87.

OLD, J. (1991). Analysis of polysemy and homography of the word "lead" in Roget's International Thesaurus. In Proceedings of the Third Midwest Artificial Intelligence and Cognitive Science Society Conference (pp. 98-102), Carbondale, IL.

PAWLAK, Z. (1982). Rough sets. International Joumal of Computer \& Information Sciences, 11, 341-356.

Pawlak, Z., Slowinski, K., \& Slowinski, R. (1986). Rough classification of patients after highly selective vagotomy for duodenal ulcer. International Journal of Man-Machine Studies, 24, 413-433.

Sedelow, W. SEDELow, S. (1992). Toward generic artificial intelligence: A different tack. In Proceedings of the Fourth Midwest Artificial Intelligence and Cognitive Science Sociery Conference (pp. 122-130). Utica, IL.

VAN DE VELDE, W. (1988). Leaming through progressive refinement. In Proceedings of the EWSL 88, Third European Working Session on Learning (pp. 211-226). Marshfield, MA: Pitman. 